\title{
General solution for scalar perturbations in bouncing cosmologies
}

\author{
V. Bozza \\ Dipartimento di Fisica "E. R. Caianiello", Università di Salerno, I-84081 Baronissi, \\ Italy. \\ Istituto Nazionale di Fisica Nucleare, Sezione di Napoli, Naples, Italy.
}

\begin{abstract}
Bouncing cosmologies, suggested by String/M-theory, may provide an alternative to standard inflation to account for the origin of inhomogeneities in our universe. The fundamental question regards the correct way to evolve the scalar perturbations through the bounce. In this work, we determine the evolution of perturbations and the final spectrum for an arbitrary (spatially flat) bouncing cosmology, with the only assumption that the bounce is governed by a single physical scale. In particular, we find that the spectrum of the pre-bounce growing mode of the Bardeen potential (which is scale-invariant in some limit, and thus compatible with observations) survives unaltered in the post-bounce only if the comoving pressure perturbation is directly proportional to the Bardeen potential rather than its Laplacian, as for any known form of ordinary matter. If some new physics acting at the bounce justifies such relation, then bouncing cosmologies are entitled to become a real viable alternative for the generation of the observed inhomogeneities. Our treatment also includes some class of models with extra-dimensions, whereas we show that bounces induced by positive spatial curvature are structurally different from all bounces in spatially flat universes, requiring a distinct analysis.
\end{abstract}

PACS numbers: 98.80.-k, 98.65.Dx, 98.80.Es, 11.25.Wx 


\section{Introduction}

The occurrence of singularities in classical General Relativity (GR) may be the symptom of the existence of a more general physical frame, where GR is embedded as a particular low curvature limit. In such a frame, that we will generically call "new physics", one may expect that singularities are cured by the existence of a natural physical cutoff, i.e. a scale where the new physics comes into play, replacing GR. Presently, the only consistent example of such a general theory is String theory, or its 11-dimensional generalization M-theory, where this cutoff is naturally provided by the string length $l_{s}$.

Following this reasoning line, in a cosmological context, it is natural to expect that the new physics would prevent the Big Bang singularity predicted by GR, allowing a continuation of the cosmological solutions before the Big Bang. This idea, firstly proposed by Veneziano and Gasperini [1], has found several alternative implementations and recurrently emerges in independent attempts to build consistent cosmological models from String-M/theories [2, 3, 4, 5]. All these models, when formulated in the Einstein frame, share the same general behavior: the universe starts from an asymptotically flat state and undergoes a contraction that pumps the space-time curvature up to the new physics scale. At this point, the new physics masters the transition to an expanding phase and the standard Friedman-Robertson-Walker (FRW) universe begins. In the present literature, such models are generically called bouncing cosmologies.

The interest in bouncing cosmological models comes from the fact that an accelerated contraction seems to solve the flatness and horizon problems as efficiently as a standard inflationary phase. Then the question opens whether a bouncing cosmology may replace standard inflation once and for all. In order to achieve this fascinating objective, a bouncing cosmology should provide its own mechanism of generation of the inhomogeneities of the universe, in such a way that all present constraints are satisfied at the same accuracy level of standard inflation. The most stringent observational constraint comes from CMB anisotropies, which require the initial power spectrum of the Bardeen potential (encoding the information on all scalar cosmological perturbations) to be (nearly) scale-invariant. Is this obtainable or not in a bouncing cosmology? The first studies in the Pre-Big Bang scenario gave a negative answer [6], indicating that the inhomogeneities must have an independent origin. For example, they can be generated in an axion-dominated era through the curvaton mechanism [].

On the other hand, the authors of the Ekpyrotic/cyclic scenario [3] claimed that a scale-invariant spectrum can be generated in a slow contraction era and transmitted across the bounce. This has risen a considerable debate [8, 9] about the correct way to describe the evolution of perturbations through a bounce, but, after several years of investigation, it seems quite difficult to draw a general conclusion, valid for all models of bouncing cosmologies.

The evolution of perturbations in a bouncing cosmology can be summarized very quickly as follows: in the pre-bounce phase, the Bardeen potential has a growing 
mode, endowed with a red spectrum (becoming scale-invariant in the limit of very slow contraction [3]) and a decaying mode with a blue spectrum (becoming scale-invariant in the limit of dust-dominated contraction [4]). After the bounce, we have two alternatives: either the spectrum of the growing mode is inherited by a constant mode in the postbounce or the growing mode simply decays in the post-bounce and some other constant mode with a more blue spectrum dominates at horizon re-entry.

If the first possibility occurs, then the Ekpyrotic/cyclic models would become a complete alternative to standard inflation. Otherwise, a curvaton mechanism or some other one must be implemented to make such models compatible with observations. All studies of specific (spatially flat) toy models, where perturbations are explicitly calculable, show that the pre-bounce growing mode decays in the post-bounce and the Bardeen potential is finally dominated by a blue spectrum, incompatible with observations [10, 11, 12, 13] (the incorrect conclusions reached in Refs. 14, 15] are revised in Ref. [13] and reconciled with all other studies; also the model presented in Ref. [16] is a particular case of the general model presented in Ref. [13, with two perfect fluids with $w_{a}=1 / 3$ and $w_{b}=5 / 3$ ). However, the investigation of specific models does not allow to infer any general conclusions, since the possibility that some still unknown new physics may intervene at the bounce, producing different effects, remains open. Indeed, bounces induced by positive spatial curvature show a different behavior, with a generic mixing between growing and decaying modes [17, 18].

The description of the evolution of scalar perturbations through a bounce has acquired an independent interest that goes beyond the Ekpyrotic/cyclic models, thanks to the mathematical complexity of the problem, which poses several questions and issues of general theoretical interest.

In this work we present a completely analytical study of scalar perturbations in spatially flat bouncing cosmologies, making the only assumption that the bounce is governed by a single physical scale. In this way, we are able to establish a univocal relation between the final spectrum of perturbations and the form of the total energymomentum tensor. We thus introduce a sort of golden rule for scalar perturbations in bouncing cosmologies.

In Sect. 2 we define the generic bouncing cosmology, stating the hypotheses that we need to deduce our general result. In Sect. 3 we elaborate our hypotheses, describing the background cosmology. In Sect. 4 we write the linear perturbation equations. In Sect. 5 we derive the general form of the effective energy-momentum tensor, including the high energy corrections driving the bounce. In Sect. 6 we compute the evolution of perturbations in the pre-bounce, in the bounce and in the post-bounce. In Sect. 7 we illustrate the general results of Sect. 6 by some numerical examples. In Sect. 8 we consider possible extensions to models with extra-dimensions and analyze the differences between bounces in spatially flat universes and bounces in spatially closed universes. Sect. 9 contains the conclusions. 


\section{The generic bouncing cosmology}

Let us start by enumerating our hypotheses:

(i) We assume that it makes sense to define a four-dimensional metric tensor, both in the GR regime and in the new physics regime. Then it is possible to write the four-dimensional Einstein equations with an effective energy-momentum tensor on the right hand side, encoding all the corrections dictated by the new physics.

(ii) The universe is homogeneous, isotropic and spatially flat, thus being described by the FRW metric.

(iii) There is a unique physical scale $\eta_{B}$ governing the bounce. We will set our units in such a way that $c=1,8 \pi G=1, \eta_{B}=1$.

(iv) In the GR regime far from the bounce $(|\eta| \gg 1)$, the energy momentum tensor is characterized by a constant equation of state and a constant speed of sound, with $p / \rho>-1 / 3$.

In Sect. 8, we will comment on models with extra-dimensions which may fit into hypothesis (i) and discuss the differences with positive curvature bounces.

Now let us work out these hypotheses. The metric, including scalar perturbations, is

$$
d s^{2}=a^{2}(\eta)\left\{(1+2 \phi) d \eta^{2}-2 B_{, i} d \eta d x^{i}-\left[(1-2 \psi) \delta_{i j}+2 E_{, i j}\right] d x^{i} d x^{j}\right\},
$$

where we have used the conformal time $\eta$. The scale factor is $a(\eta)$, while $B, E$, $\psi$ and $\phi$ are scalar perturbations.

The total energy-momentum tensor reads

$$
T^{\mu}{ }_{\nu}=\left(\begin{array}{cc}
\rho+\delta \rho & -(\rho+p) \mathcal{V}_{, i} \\
(\rho+p) \mathcal{V}_{, i} & -(p+\delta p) \delta_{i j}-\xi_{, i j}
\end{array}\right),
$$

where $\delta \rho$ is the energy density perturbation, $\delta p$ is the pressure perturbation, $\mathcal{V}$ is the velocity potential and $\xi$ is the anisotropic stress. Note that these expressions do not require GR, but are just dictated by the symmetry of the problem (hypothesis (ii)). Thus, also in the bounce regime, the new physics corrections encoded in $T^{\mu}{ }_{\nu}$ will respect this symmetry and contribute to its components, both at the background and at the perturbative level. Since this tensor stays on the right hand side of the Einstein equations, it must be compatible with the Bianchi identities, thus it must be covariantly conserved: $T_{\nu ; \mu}^{\mu}=0$.

The independent background Einstein equations and the energy-momentum conservation give the Friedman equations

$$
\begin{aligned}
& 3 \mathcal{H}^{2}=a^{2} \rho \\
& \mathcal{H}^{2}+2 \mathcal{H}^{\prime}=-a^{2} p \\
& \rho^{\prime}+3 \mathcal{H}(\rho+p)=0,
\end{aligned}
$$


where the prime denotes derivative w.r.t. $\eta$ and $\mathcal{H}=a^{\prime} / a$ is the conformal Hubble rate.

In a spatially flat universe the Null Energy Condition (NEC), which amounts to require $\rho+p>0$, must be necessarily violated during the bounce. Of course, this violation cannot be achieved by ordinary matter, but requires the intervention of the new physics. One may object that this implies the presence of ghosts at the quantum level. While the majority of explicit bouncing cosmologies in the literature indeed contain ghosts, there are at least two counterexamples. The first is the model by Gasperini, Giovannini and Veneziano [11, where the NEC violation is performed by a potential for the scalar field involving integration on the spatial volume of the universe. The second is the model by Biswas, Mazumdar and Siegel [5], where the generalized gravitational action is an analytic function of the D'Alembert operator acting on the curvature scalar without poles. In both cases there are no ghosts in the theory. In any case, the possible existence of ghosts for a finite time interval may not be a problem at all [19].

\section{Background}

Now let us determine the background evolution far from the bounce, using hypothesis (iv). In the pre-bounce we have $p=w_{-} \rho$ and in the post-bounce $p=w_{+} \rho$, with constant $w_{-}$and $w_{+}$. The restriction $w_{+}>-1 / 3$ excludes the possibility of a postbounce inflation, which would spoil the results of the bounce. On the other hand, a pre-bounce deflation, excluded by the restriction $w_{-}>-1 / 3$, would make the setting of initial conditions in the asymptotic past problematic.

We set $\eta=0$ at the bounce, defined as the time where $a^{\prime}(\eta)=0$. The bounce duration scale must be of the same order of the curvature scale, according to hypothesis (iii). Otherwise, there would be two independent scales. So, the bounce will last from $\eta_{-} \simeq-1$ to $\eta_{+} \simeq 1$.

Then, the pre-bounce background functions have the power law behavior

$$
\begin{aligned}
& a(\eta) \simeq|\eta|^{q_{-}} \\
& \mathcal{H} \simeq \frac{q_{-}}{\eta} \\
& \rho_{a} \simeq \frac{3 q_{-}^{2}}{|\eta|^{2+2 q_{-}}} \\
& q_{-}=\frac{2}{1+3 w_{-}},
\end{aligned}
$$

where we have chosen all constant of integration in such a way that the space-time curvature at the onset of the bounce $\eta \sim-1$ is of order 1 .

In the same way, we can write the post-bounce behavior of the background functions, which will be analogous to Eqs. (6)-(9) with all "-" in the subscripts replaced by "+". 


\section{Perturbations equations}

We will write the perturbations equations in terms of the following gauge invariant variables

$$
\begin{aligned}
& \Psi=\psi+\mathcal{H}\left(E^{\prime}-B\right) \\
& \zeta=\psi+\mathcal{H} \mathcal{V} \\
& \delta \rho_{\mathcal{V}}=\delta \rho-\rho^{\prime} \mathcal{V} \\
& \delta p_{\mathcal{V}}=\delta p-p^{\prime} \mathcal{V}
\end{aligned}
$$

plus the anisotropic stress $\xi$. $\Psi$ is the Bardeen potential, $\zeta, \delta \rho \mathcal{V}$ and $\delta p_{\mathcal{V}}$ are respectively the curvature, energy density and pressure perturbations on comoving hypersurfaces. In the following we will drop the subscript " $\mathcal{V}$ " from all variables. Then, from now on, $\delta \rho$ and $\delta p$ must be intended as comoving energy density and pressure perturbations.

Using the component $(0 i)$ of the Einstein equations to eliminate $\phi$, the $(00),(i j)$ and $(i i)$ components become

$$
\begin{aligned}
& \delta \rho=\frac{2}{a^{2}} \nabla^{2} \Psi \\
& \delta p-\nabla^{2} \xi=-\frac{2\left(\mathcal{H}^{2}-\mathcal{H}^{\prime}\right)}{a^{2} \mathcal{H}} \zeta^{\prime} \\
& a^{2} \mathcal{H} \xi=-\frac{\mathcal{H}^{2}-\mathcal{H}^{\prime}}{\mathcal{H}} \zeta+\frac{2 \mathcal{H}^{2}-\mathcal{H}^{\prime}}{\mathcal{H}} \Psi+\Psi^{\prime} .
\end{aligned}
$$

Some of the coefficients of these equations become singular at the bounce $(\mathcal{H}=0)$ or at the NEC violation time $\left(\mathcal{H}^{2}-\mathcal{H}^{\prime}=0\right)$. In order to check the regularity of our variables, we may resort to the regular gauge argument used in Ref. [13]. If a gauge exists where all components of the metric and energy-momentum tensor stay finite, then we can explicitly check the regularity of our gauge invariant variables, expressing them in this gauge. As shown in [13, $\Psi$ is regular while $\zeta$ is not. However, we may construct $\tilde{\zeta}=\left(\mathcal{H}^{2}-\mathcal{H}^{\prime}\right) \zeta$, where the extra factor compensates the possible divergence of $\mathcal{V}$ at the NEC violation, allowed by the fact that it is the combination $(\rho+p) \mathcal{V}$ that appears in the energy-momentum tensor and is guaranteed to be regular by the regular gauge hypothesis. $\delta \rho_{\mathcal{V}}$ has no divergences (since $\rho^{\prime}=0$ at the NEC violation time, thanks to the continuity equation), while for $\delta p_{\mathcal{V}}$ we should use the regularized variable $\delta \tilde{p}_{\mathcal{V}}=\left(\mathcal{H}^{2}-\mathcal{H}^{\prime}\right) \delta p_{\mathcal{V}}$. Dealing with regularized variables is absolutely necessary in all numerical applications, because no integrator can step through a divergence. However, for our analytical arguments, it is completely equivalent to discuss the equations (14)(16) or their regularized counterparts. We choose the unregularized variables only because they have an immediate physical interpretation, but we would get exactly the same results with the regularized variables.

Going back to our equations, Eq. (14) represents the analogous of the Poisson equation, relating the Laplacian of the gravitational potential to the perturbations in the energy density (this is not a dynamical equation). Eq. (15) is a first order equation 
in $\zeta$, which depends on the sources $\delta p$ and $\xi$. The anisotropic stress also enters as a source in the first order equation for $\Psi$ (16). It is easy to verify that the perturbations of the continuity equations give no new independent equations.

All the dynamics of scalar perturbations is contained in these three equations. In order to solve them, we need to specify the sources $\delta p$ and $\xi$ for the energy-momentum content of our universe. This is easy for the pre-bounce and post-bounce phases. In fact, if they are dominated by a perfect fluid or a scalar field with some potential, the sources are

$$
\delta p=c_{s}^{2} \delta \rho ; \quad \xi=0,
$$

with $s$ being "-" or "+" in the pre-bounce and post-bounce respectively. If the squared speed of sound $c_{s}^{2}$ is equal to $w_{s}$, we have a perfect fluid, otherwise we have a fluid with some intrinsic isocurvature mode [13]. In particular, a scalar field with a potential has always $c_{s}^{2}=1$, whatever the equation of state induced by the presence of the potential. In any case, with these sources, Eqs. (14)-(16) can be combined into a second order equation for $\zeta$ or $\Psi$, suitable for vacuum normalization. Expanding the variables in Fourier modes of wavenumber $k$ as usual, in the asymptotic past we have

$$
\zeta=C \frac{\mathcal{H}}{a \sqrt{\mathcal{H}^{2}-\mathcal{H}^{\prime}}} \sqrt{|\eta|} H_{\nu}^{(1)}\left(c_{-} k|\eta|\right),
$$

where $C$ is a normalization constant of order $1, H_{\nu}^{(1)}$ is the Hankel function of the first kind and $\nu=1 / 2-q_{-}$.

We are interested in modes that are well outside the horizon at the bounce, thus having $k \ll 1$. For such modes, in the range $-1 / k<\eta<-1$, we can use the expansion of the Hankel functions for small arguments and Eq. (15) to determine $\Psi$. Discarding all numerical factors of order one, we have two modes for each variable

$$
\begin{aligned}
& \zeta \sim k^{\nu}|\eta|^{1-2 q_{-}}+k^{-\nu} \\
& \Psi \sim k^{\nu-2}|\eta|^{-1-2 q_{-}}+k^{-\nu} .
\end{aligned}
$$

$\Psi$ is characterized by a growing mode with a red spectrum (becoming scale-invariant in the limit $q_{-} \rightarrow 0$ ) and a constant mode with a blue spectrum. $\zeta$ is characterized by a mode that is growing for $q_{-}>1 / 2$ and decaying otherwise. This mode has a spectrum that is blue for $q_{-}<2$ and red otherwise, being scale-invariant in the limit of dust-dominated pre-bounce [4]. The second mode is a blue constant mode.

\section{General form of the energy-momentum tensor of perturbations}

In order to discuss the evolution of perturbations through the bounce, we need to specify the form of the sources. In the ignorance of the new physics governing the bounce, we can still select all admissible forms of the pressure perturbation and the anisotropic stress and make a complete discussion of what can be expected at the end of the bounce.

Firstly, let us deal with the pressure perturbation, leaving aside the anisotropic stress for the moment. 
The pressure perturbation can be a generic linear combination of $\delta \rho, \Psi, \zeta$ and a certain number $n$ of additional internal variables $\delta \varphi_{i}$, with time depending coefficients. Of course, the pressure perturbation may also depend on the time and space derivatives of these variables. Now, through Eq. (14), any dependence on $\delta \rho$ can be read as an additional dependence on $\Psi$ and its derivatives.

For each internal variable it is necessary to specify an independent linear differential equation in order to close the system. We are not interested in any new spectral dependence whose origin is different from vacuum normalization of the original scalar degree of freedom. Indeed, if such spectral dependences are carried by these internal variables, they may play an important role, giving rise to additional isocurvature modes in the post-bounce, as in the model discussed in Ref. [13]. They depend on the specific model and must be carefully considered in any complete treatment. However, our question is more general and only involves the fate of the original spectrum of $\Psi$ and $\zeta$. The internal variables may acquire this spectrum only if $\zeta, \Psi$ and their derivatives explicitly appear in their equations of motion. But then their feedback on $\delta p$ would be an effective renormalization of the coefficients of $\zeta, \Psi$ and their derivatives in the expression of $\delta p$.

$\delta p$ is then just a linear combination of $\Psi, \zeta$ and their derivatives with timedependent coefficients. However, $\delta p$ is scalar under spatial rotations and thus it must contain only scalars. The time derivatives of scalars are still scalars under spatial rotation, but the spatial derivatives must be combined with themselves or other vectors of the theory in order to produce scalars. More specifically, the spatial derivatives can only appear as $\nabla^{2}$ or $A^{i} \partial_{i}$, where $A^{i}$ is a generic 3 -vector of the theory. However, since the background is homogeneous and isotropic, all 3 -vectors in the background must be zero. So, spatial derivatives can only appear in a Laplacian or a function of the Laplacian.

Summing up, the most general expression for $\delta p$ is

$$
\delta p=\frac{2}{a^{2}} \sum_{n=0}^{\infty} F_{n}\left(\eta, \partial_{\eta}\right) k^{2 n} \zeta+\frac{2}{a^{2}} \sum_{n=0}^{\infty} G_{n}\left(\eta, \partial_{\eta}\right) k^{2 n} \Psi,
$$

where we have supposed that the dependence on the Laplacians is analytic and allows a regular power expansion and we have introduced the factor $2 / a^{2}$ for later convenience. Using the Fourier mode expansion, the Laplacians have been written as $-k^{2}$. With the same arguments, a similar expression should also hold for the anisotropic stress

$$
\xi=\frac{2}{a^{2}} \sum_{n=0}^{\infty} J_{n}\left(\eta, \partial_{\eta}\right) k^{2 n} \zeta+\frac{2}{a^{2}} \sum_{n=0}^{\infty} K_{n}\left(\eta, \partial_{\eta}\right) k^{2 n} \Psi .
$$

Of course, any possible crossed dependence between $\delta p$ and $\xi$ can be easily solved at the linear level.

Then the physics of the model is completely encoded in the time-dependent operators $F_{n}\left(\eta, \partial_{\eta}\right), G_{n}\left(\eta, \partial_{\eta}\right), J_{n}\left(\eta, \partial_{\eta}\right), K_{n}\left(\eta, \partial_{\eta}\right)$. Let us make some examples.

For a single perfect fluid or a scalar field, as mentioned before, the only nonvanishing operator is $G_{1}=-c_{s}^{2}$. In the bounce induced by two perfect fluids or scalar 
fields, studied in Ref. [13] and containing the models by Peter \& Pinto-Neto [14], Finelli [15], and Allen \& Wands [12, two internal variables are present. Taking into account their dependence on $\zeta$ and $\Psi$, the final expression for the effective energymomentum tensor only contains two non-vanishing coefficients: $F_{0}$, and $G_{1}$, i.e. the pressure perturbation only depends on $\zeta$ and $k^{2} \Psi$, while the anisotropic stress is zero. The model by Gasperini, Giovannini and Veneziano [11 is simpler, since it contains no internal variables and the only non-vanishing operator is $G_{1}=4\left(\mathcal{H}^{\prime}-\mathcal{H}^{2}\right) / \varphi^{\prime 2}$, where $\varphi$ is the dilaton field in their model. Cartier's model [10] has a very involved effective energy-momentum tensor with a non-vanishing anisotropic stress. Using the expressions in the appendix of his work, we find that the covariant conservation is fulfilled only in the trivial case $\mathcal{F}=1 / 2, \alpha^{\prime}=0$, indicating that some misprint may be present in the expressions published therein. So, we renounced to identify our operators $F_{n}, G_{n}, J_{n}$ and $K_{n}$ in this otherwise interesting model.

\section{Evolution of perturbations}

In this section we shall study the evolution of scalar perturbations through all cosmological eras, making no assumptions on the specific form of the energy-momentum tensor, thus retaining all terms in the general expressions (21), (22).

In Ref. [13] an easy way to find the evolution of perturbations outside the horizon was proposed. It amounts to write the first order differential equations in their integral form and write the solution as a recursive series. The integral form of Eqs. (15)-(16) is

$$
\begin{aligned}
\Psi & =\frac{\mathcal{H}}{a^{2}}\left[\frac{c_{1}(k)}{k^{2}}+\int \frac{a^{2}\left(\mathcal{H}^{2}-\mathcal{H}^{\prime}\right)}{\mathcal{H}^{2}} \zeta d \eta+\int a^{2} \mathcal{H} \xi d \eta\right] \\
\zeta & =\left[c_{2}(k)-\int \frac{a^{2} \mathcal{H}}{2\left(\mathcal{H}^{2}-\mathcal{H}^{\prime}\right)} \delta p d \eta-\int \frac{a^{2} \mathcal{H}}{2\left(\mathcal{H}^{2}-\mathcal{H}^{\prime}\right)} k^{2} \xi d \eta\right],
\end{aligned}
$$

where $c_{1}(k)$ and $c_{2}(k)$ are two integration constants.

With these equations it is very easy to find the evolution of perturbations in any phase. In fact, using the general expressions of $\delta p$ and $\xi$, we can recast the set of equations in the following form

$$
X(\eta, k)=X^{(0)}(\eta, k)+\hat{A}(\eta, k) \int \hat{B}\left(\eta, \partial_{\eta}, k\right) X(\eta, k) d \eta,
$$

where $X=(\Psi, \zeta)$ is the vector of variables, $X^{(0)}=\left(c_{1}(k) \mathcal{H} / a^{2}, c_{2}(k)\right)$ is the vector containing the decoupled solutions and $\hat{A}(\eta, k)$ and $\hat{B}\left(\eta, \partial_{\eta}, k\right)$ are two coupling matrices that can be deduced from Eqs. (23) and (24), once we use the proper form of the sources as functions of $\zeta$ and $\Psi$. The general solution to Eq. (25) is

$$
X=\sum_{i=0}^{\infty} X^{(i)}
$$

where $X^{(i)}$ is determined by $X^{(i-1)}$ as

$$
X^{(i)}=\hat{A}(\eta, k) \int \hat{B}\left(\eta, \partial_{\eta}, k\right) X^{(i-1)}(\eta, k) d \eta .
$$


So, starting from the decoupled solution $X^{(0)}$, we can construct the full solution of the system. Of course, this only works for modes outside the horizon, where we can stop to the first few terms of the series because the higher order terms contain extra-factors of $k^{2} \ll 1$ or $k^{2} \eta^{2} \ll 1$. Let us examine the solution in each phase.

\subsection{Pre-bounce}

In the pre-bounce, the soulution assumes a very simple form. In fact, there we have $\delta p=-2 c_{-}^{2} k^{2} \Psi / a^{2}$ and $\xi=0$, while the explicit expression for all background functions in terms of powers of $\eta$ is given by Eqs. (6) -(9). So, it is very easy to perform all integrations. Discarding all numerical factors of order one, we start from the decoupled solution, which assumes the following form in the power law regime

$$
\begin{aligned}
& \Psi^{(0)}=k^{-2} c_{1}(k)|\eta|^{-1-2 q_{-}} \\
& \zeta^{(0)}=c_{2}(k) ;
\end{aligned}
$$

the first recursion gives

$$
\begin{aligned}
& \Psi^{(1)}=c_{2}(k) \\
& \zeta^{(1)}=c_{1}(k)|\eta|^{1-2 q_{-},}
\end{aligned}
$$

and the second recursion gives

$$
\begin{aligned}
& \Psi^{(2)}=c_{1}(k)|\eta|^{1-2 q_{-}} \\
& \zeta^{(2)}=k^{2} c_{2}(k)|\eta|^{2} .
\end{aligned}
$$

We can see that the second recursion gives the same modes already present in the decoupled solution, multiplied by $k^{2} \eta^{2}$. Since we are considering the evolution outside the horizon, this factor is much smaller than one and we can safely neglect the second and all higher order recursions. We are thus left with

$$
\begin{aligned}
& \Psi_{-} \sim k^{-2} c_{1}(k)|\eta|^{-1-2 q_{-}}+c_{2}(k) \\
& \zeta_{-} \sim c_{1}(k) \eta^{1-2 q_{-}}+c_{2}(k) .
\end{aligned}
$$

Comparing this solution with the expansion of the Hankel functions for small arguments, given by Eqs. (19) and (20), we can make the identifications $c_{1}(k)=k^{\nu}, c_{2}(k)=k^{-\nu}$, recovering the two integration constants in terms of the vacuum normalization.

\subsection{Bounce}

Now let us turn to the core of our work. It must be said that the specific shape of the solution during the bounce is not our concern, since we just want to know the final spectrum of scalar perturbations when they reenter the horizon. However, as we shall see in the next subsection, in order to construct the post-bounce solution, we need to evaluate the integrals appearing in Eq. (27) in a domain covering the whole bounce phase, which ranges from $\eta \simeq-1$ to $\eta \simeq 1$ in our normalized units. It turns out that 
the evaluation of the bounce integrals is very easy, since the $k$ and $\eta$ dependences are always factored. In fact, the generic integral has the form

$$
\int_{-1}^{1} f(k, \eta) d \eta=f_{k}(k) \int_{-1}^{1} f_{\eta}(\eta) d \eta=f_{k}(k) .
$$

The last equality is crucial. It comes from the fact that the only scales in our problem are $\eta, k$ and the bounce scale $\eta_{B}$ that we have set to 1 . Since we have factored the $k$ dependence, the only scale left in $f_{\eta}$ is $\eta$ itself. But integrating over $\eta$ from -1 to 1 , whatever the dependence of the primitive function on $\eta$, the final result will lose the only scale it contained, and would thus be of order 1 . It cannot depend on any other scale, unless the bounce is actually governed by two or more scales. In that case, we cannot solve the integrals in such a trivial way, except in some special limits.

Since we are going to use several integrals over the bounce phase in the next section, we give their explicit derivation in Appendix A.

\subsection{Post-bounce}

In order to build the solution after the bounce, at every recursion we must evaluate integrals of the form

$$
\int_{-\infty}^{\eta} f(k, \eta) d \eta
$$

The integration domain can be split in three parts, covering the pre-bounce, the bounce and the post-bounce phase, respectively. We thus have

$$
\int_{-\infty}^{\eta} f(k, \eta) d \eta=\int_{-\infty}^{-1} f(k, \eta) d \eta+\int_{-1}^{1} f(k, \eta) d \eta+\int_{1}^{\eta} f(k, \eta) d \eta .
$$

For the pre-bounce integrals, it is sufficient to use the pre-bounce solutions evaluated at $\eta \simeq-1$. For the bounce integrals, we will exploit the explicit forms calculated in Appendix A according to the prescription given in the previous subsection. The postbounce integrals can be easily evaluated using the asymptotic background solution in the post-bounce phase, where all background functions are replaced by powers of $\eta$. For a generic function $f(k, \eta)=f_{k}(k)|\eta|^{s}$, we have

$$
\int_{1}^{\eta} f_{k}(k)|\eta|^{s} d \eta \simeq f_{k}(k)\left[|\eta|^{s+1}+1\right]
$$

where we have discarded all numerical factors of order 1, as usual.

We are now ready to construct the post-bounce solution. Once more, we start from

the decoupled solution, that in the post-bounce era again assumes the explicit power-law form

$$
\begin{aligned}
& \Psi^{(0)}=k^{\nu-2}|\eta|^{-1-2 q_{+}} \\
& \zeta^{(0)}=k^{-\nu} .
\end{aligned}
$$


Then, the first recursion gives

$$
\begin{aligned}
& \Psi^{(1)}=|\eta|^{-1-2 q_{+}}\left[k^{-\nu}\left(|\eta|^{1+2 q_{+}}+1\right)+\int_{-1}^{1} f_{\eta}(\eta) \zeta^{(0)}+\int_{-1}^{1} f_{\eta}(\eta) \xi^{(0)} d \eta\right] \\
& \zeta^{(1)}=k^{\nu}\left(|\eta|^{1-2 q_{+}}+1\right)+\int_{-1}^{1} f_{\eta}(\eta) \delta p^{(0)} d \eta+k^{2} \int_{-1}^{1} f_{\eta}(\eta) \xi^{(0)} d \eta
\end{aligned}
$$

where the bounce integrals can be read from the appendix.

The second recursion gives

$$
\begin{aligned}
& \Psi^{(2)}=|\eta|^{-1-2 q_{+}}\left[k^{\nu}\left(|\eta|^{1+2 q_{+}}+|\eta|^{2}+1\right)\right. \\
& +\left(\int_{-1}^{1} f_{\eta}(\eta) \delta p^{(0)} d \eta+k^{2} \int_{-1}^{1} f_{\eta}(\eta) \xi^{(0)} d \eta\right)\left(|\eta|^{1+2 q_{+}}+1\right) \\
& \left.+\int_{-1}^{1} f_{\eta}(\eta) \zeta^{(1)} d \eta+\int_{-1}^{1} f_{\eta}(\eta) \xi^{(1)} d \eta\right] \\
& \zeta^{(2)}=k^{-\nu+2}\left(|\eta|^{1-2 q_{+}}+|\eta|^{2}+1\right) \\
& +k^{2}\left(\int_{-1}^{1} f_{\eta}(\eta) \zeta^{(0)}+\int_{-1}^{1} f_{\eta}(\eta) \xi^{(0)} d \eta\right)\left(|\eta|^{1-2 q_{+}}+1\right) \\
& +\int_{-1}^{1} f_{\eta}(\eta) \delta p^{(1)} d \eta+k^{2} \int_{-1}^{1} f_{\eta}(\eta) \xi^{(1)} d \eta .
\end{aligned}
$$

No further recursions are needed, since all new terms are negligible for superhorizon perturbations. At the end, according to Eq. (26) we can sum up all contributions to build the full post-bounce solution. A careful analysis reveals that almost all terms coming from the bounce integrals are of the same order of other terms already present or are negligible for perturbations outside the horizon at the bounce. Then, the final post-bounce solution takes the amazingly simple form

$$
\begin{aligned}
\Psi_{+} & \sim k^{\nu-2}|\eta|^{-1-2 q_{+}}+k^{-\nu}+k^{\nu}+G_{0} k^{\nu-2} \\
\zeta_{+} & \sim k^{\nu} \eta^{1-2 q_{+}}+k^{-\nu}+k^{\nu}+G_{0} k^{\nu-2},
\end{aligned}
$$

where the constant $G_{0}$ is set to 1 if the corresponding operator defined in Eq. (21) is non-vanishing; otherwise we have $G_{0}=0$. In other words, this term is present only if $\delta p$ is directly proportional to $\Psi$ or its time derivatives, without $k^{2}$ factors, during the bounce.

\subsection{Discussion}

The whole history of perturbations in a bouncing cosmology is contained in Eqs. (19) and (20) for the pre-bounce phase, and (46), (47) for the post-bounce phase.

Let us start our discussion from the pre-bounce. The Bardeen potential in the prebounce is dominated by the growing mode $k^{\nu-2}|\eta|^{-1-2 q_{-}}$, endowed with a red spectrum 
becoming scale-invariant in the limit of slow contraction $q \rightarrow 0$. This mode comes from the decoupled solution of Eq. (16), going as $\mathcal{H} / a^{2}$ and is destined to decay in the post-bounce phase, where it takes the form $k^{\nu-2}|\eta|^{-1-2 q_{+}}$in Eq. (46). There is also a subdominant constant mode $k^{-\nu}$, which plays no role in this phase. $\zeta$ couples to $\Psi$ through a Laplacian and thus has a mode going as $k^{\nu}|\eta|^{1-2 q_{+}}$in Eq. (19), where the potentially scale-invariant spectrum is multiplied by a $k^{2}$ factor. This mode dominates for a fast contraction $q_{-}>1 / 2$ and is subdominant w.r.t. the constant mode $k^{-\nu}$, coming from the decoupled solution of Eq. (15), in the case of slow contraction $q_{-}<1 / 2$.

Now let us examine the post-bounce phase. The role of the bounce is to upset the scalar perturbations for a finite time and to determine the initial conditions for the postbounce phase. Immediately after the bounce, in Eq. (46) we have the decoupled mode $k^{\nu-2}|\eta|^{-1-2 q_{+}}$, carrying the potentially scale-invariant spectrum, two constants modes $k^{\nu}$ and $k^{-\nu}$ that are always present with a blue spectrum, and possibly one more constant mode with the $k^{\nu-2}$ spectrum. This additional mode is present only if the comoving pressure perturbation is proportional to $\Psi$ or its time derivatives without Laplacians. Recalling that we are in the limit $k \ll 1$, this constant mode will dominate undisturbed until horizon re-entry and will determine the spectrum of scalar perturbations seeding the inhomogeneities of the universe.

In the other case (i.e. if $\delta p$ is related to $\Psi$ only through Laplacians), the Bardeen potential is initially dominated by its decaying mode. But at horizon re-entry, this mode has decayed by a factor $\eta_{r e}^{-1-2 q_{+}}$, where $\eta_{r e}$ is the time of horizon re-entry. Setting $\eta_{r e} \simeq k^{-1}$, we have different possibilities. After a slow contraction in the pre-bounce phase, the larger constant mode is $k^{-\nu}$. Then, the decaying mode will still dominate only if $q_{+}<q_{-}$, i.e. if the expansion in the post-bounce is slower than the pre-bounce contraction. Otherwise, the constant mode dominates. However, in a realistic cosmology the relevant modes for CMB enter the horizon in the radiation or dust-dominated era, with $q_{+}=1$ or 2 , respectively. Then, the spectrum would be totally determined by the blue constant mode $k^{-\nu}$, that in the very slow contraction limit $q_{-} \ll 1$ gives a spectral index $n_{s}=3$. The final possibility is that the universe has undergone a fast pre-bounce contraction $q_{-}>1 / 2$, so that the largest constant mode is $k^{\nu}$. In this case, the decaying mode dominates when the expansion is slow $\left(q_{+}<1 / 2\right)$, while is negligible otherwise.

The modes dominating at horizon re-entry in all cases are summarized by the following diagram

$$
\left\{\begin{array}{l}
G_{0}=1 \Longrightarrow \Psi \sim k^{\nu-2} \\
G_{0}=0\left\{\begin{array}{l}
q_{-}<1 / 2\left\{\begin{array}{l}
q_{+}<q_{-} \Rightarrow \Psi \sim k^{\nu-2} \eta^{-1-2 q_{+}} \\
q_{+}>q_{-} \Rightarrow \Psi \sim k^{-\nu}
\end{array}\right. \\
q_{-}>1 / 2\left\{\begin{array}{l}
q_{+}<1 / 2 \Rightarrow \Psi \sim k^{\nu-2} \eta^{-1-2 q_{+}} \\
q_{+}>1 / 2 \Rightarrow \Psi \sim k^{\nu}
\end{array}\right.
\end{array}\right.
\end{array}\right.
$$

At the same time, we see that there is a direct correspondence between the modes present in $\Psi$ and those in $\zeta$, so that an analogous discussion is valid also for $\zeta$. It is interesting to note that a dependence on $\Psi$ or its time derivatives in the anisotropic stress 
does not produce any sizeable effects on the scalar perturbations. The anisotropic stress enters without Laplacians in the equation for $\Psi$, where, at most, it may renormalize the amplitude of the decaying mode. In the equation for $\zeta$ it comes through a Laplacian, thus being completely ineffective.

A very important caveat to keep in mind is that the presence of a dependence on $\Psi$ in $\delta p$ is just a necessary condition for the persistence of the $k^{\nu-2}$ spectrum in a post-bounce constant mode. In fact, bounces with particular symmetries may bring to special cancellations in the integrals, erasing the lowest order contributions to the constant mode and leaving space to higher order (most blue) contributions.

Another possibility is that the internal variables of the new physics at the bounce may provide new modes characterized by alternative spectra with a different physical origin w.r.t. quantum fluctuations in the initial vacuum state. These modes may contribute to the constant mode at the end of the bounce, providing another possible alternative source for phenomenologically acceptable scalar perturbations. However, in such case, the phenomenology would completely rely on the high energy physics at the bounce, whereas it would be preferable that the final spectrum is as independent as possible of the unknown bounce physics.

Our conclusions seem quite similar to those reached by Durrer and Vernizzi in Ref. [9], where the bounce is replaced by a spacelike hypersurface and the evolution of perturbations through the bounce is determined by Israel conditions. In fact, they find that the persistence of the growing mode of the Bardeen potential in the post-bounce era is very general, while its decay occurs only if the bounce hypersurface coincides with the uniform energy density hypersurface, which corresponds to force the surface tension to be proportional to $k^{2} \Psi$. Reinterpreting the surface tension as an integral of the pressure perturbation during the bounce, this would correspond to tune $G_{0}=0$ in our expression for $\delta p$. Thus, the two approaches seem in complete agreement.

\section{Numeric testing on toy models}

Our golden rule for scalar perturbations through bouncing cosmologies is based on a fully analytical derivation. However, it is useful to visualize the main conclusions on some toy models of bouncing cosmologies, so that they can be touched "by hand". In order to make things as simple as possible, we have considered a bounce with two perfect fluids: the first dominating the pre-bounce and post-bounce eras and the second, being characterized by negative energy density, representing the new physics effective tensor inducing the bounce. For a bounce with two perfect fluids, the Friedman equations are also integrable in terms of hypergeometric functions [15], though we will not use the analytical solutions. So, in this section, $\rho_{a}$ is the energy density of the first fluid and $\rho_{b}$ is the energy density of the second fluid. The two fluids have equations of state $p_{a}=w_{a} \rho_{a}, p_{b}=w_{b} \rho_{b}$, with $w_{a}<w_{b}$, in order to let the secondary fluid become negligible far from the bounce. According to Eq. (9), we have $q_{-}=q_{+}=q$. A slow contraction followed by a slow expansion $(q<1 / 2)$ takes place for $w_{a}>1$, while a fast 
contraction followed by a fast expansion $(q>1 / 2)$ takes place when $w_{a}<1$. As for all other models already present in the literature, in this model the post-bounce phase is the time-reversal of the pre-bounce phase. We are thus unable to directly investigate a fast contraction followed by a slow expansion or a slow contraction followed by a fast expansion. Such asymmetric bouncing cosmologies require the implementation of decay of the pre-bounce matter and creation of new matter at the bounce, which would make the model much more complicated, though not impossible to treat.

After the background is determined, we have to specify the form of the energymomentum tensor for perturbations. We choose

$$
\begin{aligned}
& \delta p=-\frac{2}{a^{2}} w_{a} k^{2} \Psi+3\left(w_{b}-w_{a}\right)\left(\rho_{b}+p_{b}\right) \zeta+G_{0}\left(\rho_{b}+p_{b}\right) \Psi \\
& \xi=0 .
\end{aligned}
$$

In the comoving pressure perturbation $\delta p$ we have included three terms. The first one is the classical adiabatic perturbation of the first fluid, which is necessary to reproduce the ordinary physics in the pre-bounce and post-bounce phases. The other two terms are multiplied by the density of the secondary fluid, which becomes negligible far from the bounce. The term proportional to $\zeta$ is the same that one would have by the requirement that the perturbations in the secondary fluid vanish in the gauge $\psi=0$. This choice is not essential and has been made just for computational reasons. The last term is a "new physics" term directly proportional to $\Psi$. We will switch it on or off by setting $G_{0}=1$ or $G_{0}=0$, respectively. Here we only present a model with vanishing anisotropic stress, as this plays no role in the final result, apart from a renormalization of some modes. Of course, we have checked that this is actually the case, testing some models (not to be presented here) with non-vanishing anisotropic stress at the bounce.

With this choice for the energy-momentum tensor of perturbations, the equations of motion are simply

$$
\begin{aligned}
\tilde{\zeta}^{\prime}+\left(1+3 w_{a}\right) \mathcal{H} \tilde{\zeta} & =-w_{a} \mathcal{H} k^{2} \Psi+\frac{G_{0}}{2} a^{2} \mathcal{H}\left(\rho_{b}+p_{b}\right) \Psi \\
\Psi^{\prime}+\frac{2 \mathcal{H}^{2}-\mathcal{H}^{\prime}}{\mathcal{H}} \Psi & =\frac{\tilde{\zeta}}{\mathcal{H}} .
\end{aligned}
$$

As anticipated before, it is safer to use $\tilde{\zeta}=\left(\mathcal{H}^{2}-\mathcal{H}^{\prime}\right) \zeta$ in all numerical applications, since this variable is guaranteed to be regular throughout the bounce if any regular gauge exists.

Imposing the vacuum normalization as the initial condition, our toy model is well defined and we can choose different values of $w_{a}, w_{b}$ and $G_{0}$ in order to test the conclusions reached analytically. The results are summarized in Fig. 1, where we have plotted $\log |\Psi|$ and $\log |\zeta|$ as functions of $\operatorname{Sign}[\eta] \log a$, which is now a common practice for the visualization of the behavior of perturbations in bouncing cosmologies [12, 13].

For Fig. 1a, we have chosen $w_{a}=1.5$ (implying $q=0.36$ ), $w_{b}=2.5$ and $G_{0}=0$. For Fig. 10, we have $w_{a}=1 / 3$ (implying $q=1$ ), $w_{b}=1$ and $G_{0}=0$. For Fig. 11, we have $w_{a}=1.5, w_{b}=2.5$ and $G_{0}=1$. These three cases illustrate almost everything we can expect in a bouncing cosmology. The spectral index $n_{s}$ of the perturbations can be 
(a)

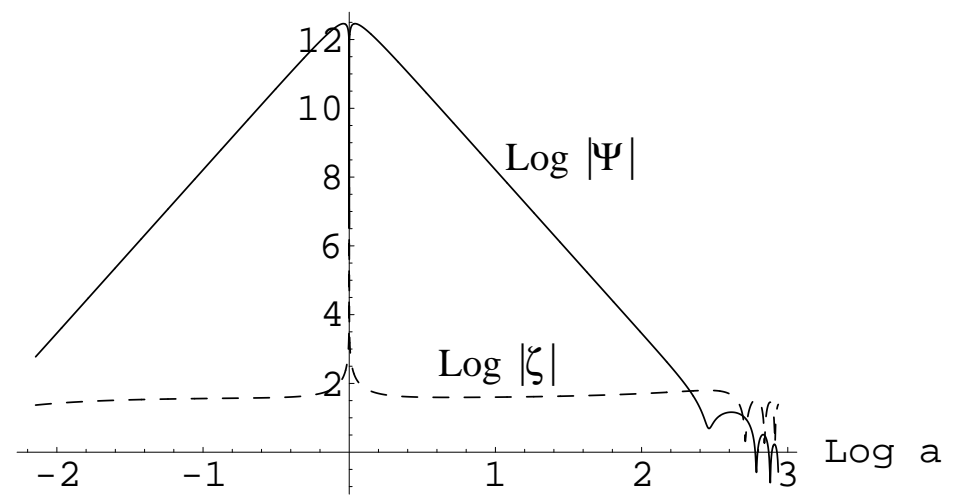

(b)

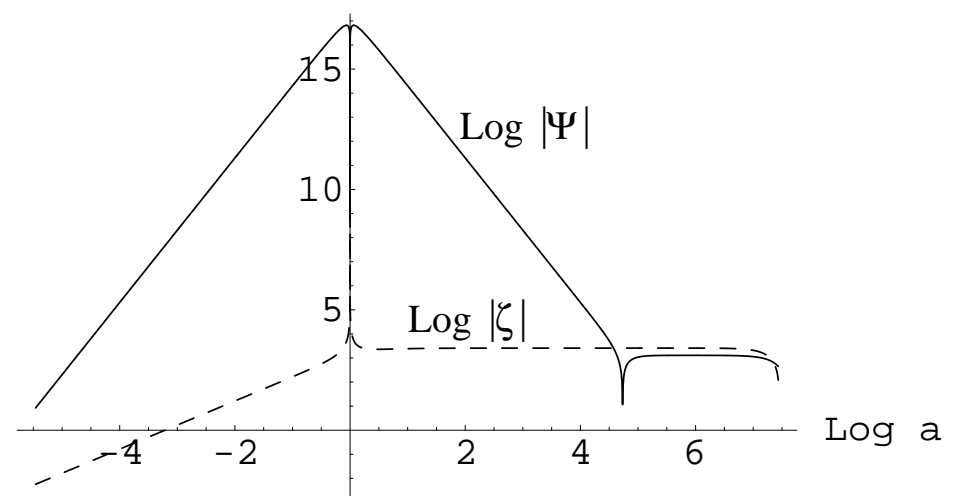

(c)

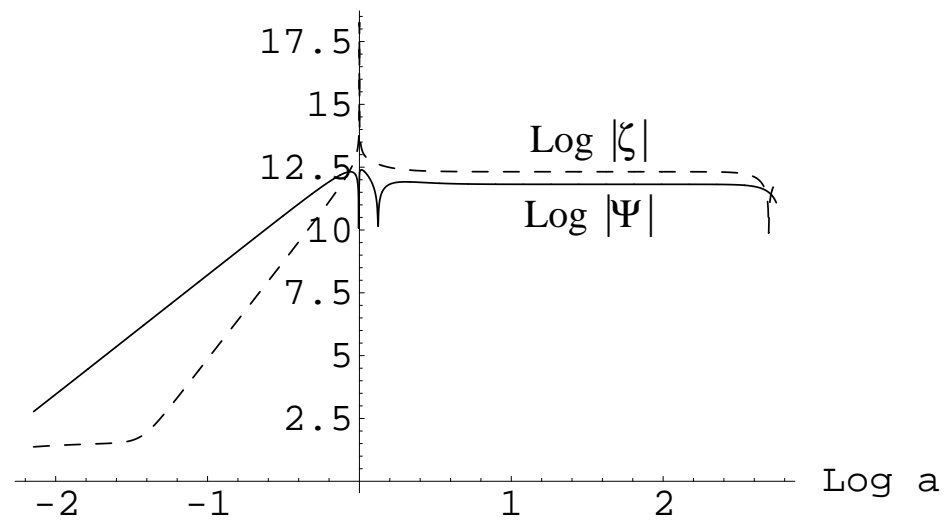

Figure 1. The three possible behaviors of scalar perturbations in bouncing cosmologies. (a) $\delta p \sim k^{2} \Psi$, with $q<1 / 2$ (slow contraction and slow re-expansion); (b) $\delta p \sim k^{2} \Psi$, with $q>1 / 2$ (fast contraction and fast re-expansion); (c) $\delta p \sim \Psi$, with any value of $q$. 


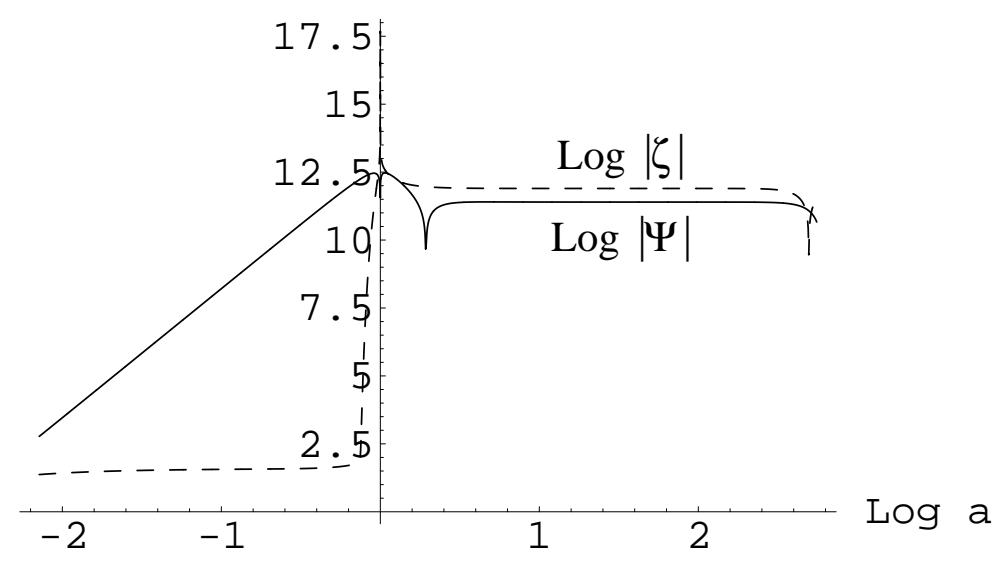

Figure 2. The same case as Fig. 1f with a different relation between $\delta p$ and $\Psi$. Here we have chosen $\delta p \sim\left(\rho_{b}+p_{b}\right) e^{1 / \rho_{b}} \Psi$.

evaluated at any time, evolving at least two Fourier modes with different $k$ and fitting the power law $k^{n_{s}-1}$. The exponent of the $\eta$-dependence is extracted by the logarithmic time derivative of the modulus of the perturbation. In this way we can compare the analytical predictions for the $k$ and $\eta$ dependences with the numerical results in all phases.

For the pre-bounce, we just have to distinguish $q<1 / 2$ (slow contraction) from $q>1 / 2$ (fast contraction). In both cases, $\Psi$ is dominated by the growing mode with the red spectrum $k^{\nu-2}|\eta|^{-1-2 q_{-}}$, as can be seen in all three plots in Fig. 1, $\zeta$ is dominated by the constant mode $k^{-\nu}$ in a slow contraction (Fig. 19) and by the growing mode $k^{\nu}|\eta|^{1-2 q_{-}}$in a fast contraction (Fig. 1b). However, when $G_{0}=1$, we see in Fig. 1p that the pre-bounce constant mode (we are in a slow contraction) is taken over by a mode going as $k^{\nu-2}|\eta|^{-2-3\left(1+w_{b}\right) q_{-}}$at some time before the bounce. This mode is induced by the direct coupling of $\zeta$ to $\Psi$ that starts to be effective during the approach to the bounce. In fact, in the model presented here, even if the term proportional to $\Psi$ in $\delta p$ is suppressed by $\left(\rho_{b}+p_{b}\right), \Psi$ grows so large that the $G_{0}$-mode starts to dominate already some time before the bounce. Indeed, choosing a function going to zero faster than $\left(\rho_{b}+p_{b}\right)$ far from the bounce, we can delay the growth of $\zeta$ as much as we like. For example, choosing $\left(\rho_{b}+p_{b}\right) e^{1 / \rho_{b}}$, the growth of $\zeta$ practically starts right at the bounce, but the post-bounce result is essentially unchanged, as shown in Fig. 2.

The post-bounce scalar perturbations essentially depend on the presence of a direct proportionality between $\delta p$ and $\Psi$. If we set $G_{0}=1$, we see from Fig. 11 that immediately after the bounce both $\zeta$ and $\Psi$ are dominated by a constant mode carrying the pre-bounce spectrum of $\Psi$, i.e. $k^{\nu-2}$. We want to stress that this outcome is completely independent of the expansion and contraction rates and of the specific form of the background function that appears in the coupling of $\delta p$ to $\Psi$. Choosing $\left(\rho_{b}+p_{b}\right)$, $\left(\rho_{b}+p_{b}\right) e^{1 / \rho_{b}}$ or any other function gives exactly the same result, apart from numerical factors of order 1 . 
If the $G_{0}$-mode is completely absent, then the pre-bounce growing mode of the Bardeen potential is entirely converted into a decaying mode, leaving no trace in the constant modes. Then, at horizon re-entry it may still dominate or it may be negligible w.r.t. some constant mode, depending on the contraction and expansion rates. In Fig. 17 we have a slow contraction followed by an expansion with the same rate. We see that at horizon re-entry the decaying mode of $\Psi$ is of the same order as the constant mode of $\zeta$, in agreement with the diagram (48), where this case is marginal between the dominance of $k^{-\nu}$ and the dominance of the decaying mode. In Fig. Ib we have a fast contraction followed by an expansion with the same rate. In agreement with the diagram (48), the decaying mode is taken over by a constant mode with a spectrum $k^{\nu}$.

\section{Remarks on bounces induced by spatial curvature and extra-dimensions}

In hypothesis (ii) we have excluded the presence of spatial curvature. Indeed, this would introduce another scale in our problem and in order to be compatible with hypothesis (iii), we have two possibilities.

The first is that the curvature scale is very low compared to the bounce scale. Then the universe is practically spatially flat and we can neglect the spatial curvature to follow the treatment of this paper.

The second is that the curvature itself determines the bounce so that the curvature scale at the bounce is actually what we have called $\eta_{B}=1$. Spatial curvature may induce a cosmological bounce only if it is positive, because it enters the Friedman equation as a perfect fluid with negative energy density and equation of state $w=-1 / 3$. The remaining source-matter may keep satisfying $\rho+p>0$, since the effective NEC violation is performed by the spatial curvature. In order to let the curvature become negligible before and after the bounce, we need $\ddot{a}>0$, i.e. decelerated contraction and accelerated expansion. For example, the pre-bounce and the post-bounce phases may be dominated by sources with equations of state $w<-1 / 3$. This contradicts our hypothesis (iv) and the initial conditions would be problematic without another early transition from an accelerated contraction in the far pre-bounce. This possibility was considered by several authors [17] as a toy model for a bouncing cosmology.

However, even relaxing hypothesis (iv), there is a structural difference between a bounce induced by spatial curvature and all other bounces with negligible spatial curvature scale. In fact, the Laplace-Beltrami operator in a positively curved space has a discrete spectrum $k^{2}=n(n+2)$ (recall that the curvature scale at the bounce has been set to 1$)$. Then, all modes are in the situation $k>1$, that means that all modes are inside the horizon at the bounce. Then our recursive solution of the integral equation would require an infinite number of terms and is completely useless. In practice, all modes re-enter the horizon during the pre-bounce deflation and oscillate in the approach to the bounce. During the inflationary phase after the bounce, they exit the horizon again, stopping the oscillations. This behavior has been put in evidence by Deruelle [18, who has described the oscillations analytically by hypergeometric functions. Then, it is not 
surprising that mode mixing after the oscillations is a generic outcome of such scenarios.

We have to conclude that bounces with positive curvature have a substantially different physics and cannot be used as a step to investigate bounces in spatially flat universes. Then it is difficult to believe that they could be useful for any physically motivated models.

However, there is one remarkable feature of spatial curvature that deserves to be mentioned. We have already noted that spatial curvature behaves as a perfect fluid with equation of state $p=-\rho / 3$ at the background level. At the linear perturbation level, we may try to recast all equations in such a way that they look like Eqs. (14)-(16) with some effective energy-momentum tensor containing the curvature terms. After some manipulation, we find the following form for the perturbations induced by the spatial curvature in the comoving gauge

$$
\begin{aligned}
& \delta \rho=-\frac{6}{a^{2}} \Psi \\
& \delta p=\frac{2}{a^{2}} \Psi+\frac{2}{a^{2} \mathcal{H}}\left(\Psi^{\prime}-\zeta^{\prime}\right)-\frac{1}{a^{2} \mathcal{H}} k^{2}(\Psi-\zeta) \\
& \xi=\frac{1}{a^{2} \mathcal{H}^{2}}(\zeta-\Psi) .
\end{aligned}
$$

Spatial curvature thus represents a very interesting example of some "new physics" with a pressure perturbation directly proportional to $\Psi$ and its time derivative without the Laplacian. So, even if spatial curvature is not useful for realistic bouncing cosmologies by itself, it encourages the conjecture that the new physics driving the bounce may contain terms directly proportional to the Bardeen potential in the effective energy-momentum tensor of perturbations.

Perhaps an even more interesting surprise comes out from extra-dimensions. In some particular cases, in fact, it is possible to recast D-dimensional field equations in the form of 4-dimensional Friedman equations with some effective energy-momentum tensor containing the dynamics of the extra-dimensions. One example is the case in which the spacetime is factored into a 4-dimensional FRW metric and an internal homogeneous manifold, where none of the metric components depends on the coordinates spanning the internal space. For example, consider a five-dimensional spacetime with the metric

$$
\begin{aligned}
d s^{2}= & a^{2}(\eta)\left\{(1+2 \phi) d \eta^{2}-2 B_{, i} d \eta d x^{i}-\left[(1-2 \psi) \delta_{i j}+2 E_{, i j}\right] d x^{i} d x^{j}\right\} \\
& -b^{2}(\eta)(1+2 \Gamma) d y^{2}-2 W d t d y
\end{aligned}
$$

with the assumption that all scalar perturbations do not depend on the internal coordinate $y$ (i.e. we are only including zero modes and neglecting excitations of the extra-dimensions). This metric was used by Battefeld, Patil and Brandenberger [20] to study bounces with extra-dimensions. It is easy to show that the background equations have the form of 4-dimensional Friedman equations with an effective contribution to the total energy-momentum tensor coming from the extra-dimension

$$
\rho_{\text {extra }}=-3 \mathcal{H} a^{-2} \frac{b^{\prime}}{b}, \quad p_{\text {extra }}=a^{-2}\left(\frac{b^{\prime \prime}}{b}+\mathcal{H} \frac{b^{\prime}}{b}\right),
$$


where the (yy) component of the Einstein equations has been used to eliminate $T^{5}{ }_{5}$.

In the same way, examining the perturbations equations, we can re-obtain Eqs. (14)-(16) with effective $\delta \rho_{\text {extra }}, \delta p_{\text {extra }}$ and $\xi_{\text {extra }}$ containing terms depending on $\Psi, \zeta \Gamma$ and their derivatives ( $W$ decouples from other perturbations). Thus, $\Gamma$ plays the role of internal variable of the model. Its dynamical equation is given by the perturbed (yy) component, with $\delta T^{5}$ as a source.

We will not present the complete expressions of the perturbed effective energymomentum tensor here, but the important lesson is that the presence of terms directly proportional to $\Psi$ in $\delta p$ is generic in this class of models as well. Then, bounces with extra-dimensions may be able to transfer the pre-bounce spectrum of $\Psi$ to the postbounce. This is in agreement with the conclusions of Ref. [20].

The fact that extra-dimensions perturbations contribute to the pressure perturbation was already noted by Giovannini [21]. Coherently with the behavior of scalar perturbations, he also finds that vector perturbations in multi-dimensional bouncing universes preserve the red spectrum acquired in the contraction phase, in contrast with 4-dimensional bouncing universes, where the red spectrum is only transferred to the decaying mode [22].

Nevertheless, a complete analysis of scalar perturbations in multi-dimensional bouncing universes that also takes into account non-zero modes is still missing. Their relevance certainly deserves some investigation, but they cannot fit into the effective four-dimensional picture presented in this paper. Further extensions of our methodology are certainly possible.

\section{Conclusions}

In this paper, we have presented the general solution for scalar perturbations in bouncing cosmologies, i.e. models of the universe where the present expansion is preceded by a contraction phase. This solution has been derived solving integral equations where the only assumption on the bounce is that it depends on a single physical scale. The "new physics" ruling the bounce has been encoded in an effective energy-momentum tensor, for which we have made no a priori assumption.

The final spectrum of the Bardeen potential in the expansion phase depends on the presence or absence in the new physics energy-momentum tensor of a direct relation between the comoving pressure perturbation and the Bardeen potential, not mediated by a Laplacian, as in all known forms of ordinary matter. If such term is present, the pre-bounce growing mode of the Bardeen potential persists in the post-bounce constant mode and it is possible to have a phenomenologically acceptable scale-invariant spectrum in Ekpyrotic-like cosmologies (i.e. models with very slow contraction in the pre-bounce). If such term is absent, the pre-bounce growing mode of the Bardeen potential decays in the post-bounce and becomes negligible w.r.t. a blue constant mode, unless the post-bounce expansion is slower than the pre-bounce contraction.

The negative conclusion reached by all regular models of 4-dimensional spatially 
flat bounces studied up to now is due to the absence of such dependence in the energymomentum tensor. Then, in order to support our conclusion, we have presented a numerical study of a toy model with $\delta p \propto \Psi$ which transfers the spectrum of the prebounce growing mode of $\Psi$ to the post-bounce growing mode.

Bounces induced by spatial curvature are structurally different from spatially flat bounces, since all modes are inside the horizon for some time interval centered at the bounce. Our treatment cannot be applied to this distinct category of bounces, whose study is anyway useless for the understanding of bounces in spatially flat cosmologies. However, spatial curvature provides a remarkable example of an effective energymomentum tensor where the pressure perturbation is proportional to the Bardeen potential without Laplacians. The same key property is also possessed by models of bounces with extra-dimensions where the dependence on the coordinates spanning the internal space is neglected. These multi-dimensional models can fit into our

effective four-dimensional scheme. However, before drawing a positive conclusion, a more rigorous and complete treatment (taking into account non-zero modes) should be envisaged for multi-dimensional bounces.

It thus seems not impossible to conjecture a form for the new physics driving the bounce that realizes the Ekpyrotic dream. The problem now is not in matching conditions or any technical issues about the evolution of perturbations, which are now definitively settled by this work. The missing ring of the chain is just the existence of some explicit model with the required property of the effective energy-momentum tensor and that is physically motivated by a consistent high energy theory like string or M-theory. When this explicit model is found, then it will be possible to say that a real alternative to standard inflation exists.

On the other hand, a more prudent attitude suggests the possibility that there might exist some important physical reasons precluding the possibility that an effective energymomentum tensor of the required form is allowed at the bounce and then the way for bouncing cosmologies would be definitively barred. However, it seems very hard to us to find any physical reason to forbid an effective pressure perturbation directly proportional to the Bardeen potential. Moreover, the explicit examples of spatial curvature and extra-dimensions seem to indicate that this possibility is actually open for high energy theories.

\section{Acknowledgments}

I am very grateful to Gabriele Veneziano for encouraging me to complete this work and for illuminating discussions. I also thank the CERN Theory Unit for hospitality. This work was partially funded by Centro studi e ricerche "Enrico Fermi", Rome, Italy. I also acknowledge useful discussions with Shini Tsujikawa, Ghazal Geshnizjani and Thirtabir Biswas. 


\section{Appendix A. Bounce integrals}

The calculation of all bounce integrals according to the prescription of Sect. 6.2 is straightforward, though a bit boring. Though we need to calculate a relatively large number of terms for the second recursion, luckily most of them are similar to other terms coming from the pre-bounce or post-bounce phases, so that they are irrelevant for the final post-bounce spectrum.

The starting point is the decoupled solution

$$
\begin{aligned}
& \Psi^{(0)}=k^{\nu-2} \frac{\mathcal{H}}{a^{2}} \\
& \zeta^{(0)}=k^{-\nu} .
\end{aligned}
$$

Note that we do not specify the shape of $a$ and $\mathcal{H}$ during the bounce phase. Then, indicating by $f_{\eta}(\eta)$ a generic function of $\eta$, the integrals involved in the first recursion are

$$
\begin{aligned}
& \int_{-1}^{1} f_{\eta}(\eta) \zeta^{(0)} d \eta=k^{-\nu} \\
& \int_{-1}^{1} f_{\eta}(\eta) \xi^{(0)} d \eta=\sum_{n=0}^{\infty}\left(J_{n} k^{-\nu+2 n}+K_{n} k^{\nu-2+2 n}\right) \\
& \int_{-1}^{1} f_{\eta}(\eta) \delta p^{(0)} d \eta=\sum_{n=0}^{\infty}\left(F_{n} k^{-\nu+2 n}+G_{n} k^{\nu-2+2 n}\right),
\end{aligned}
$$

where the first and the second integrals enter $\Psi^{(1)}$, while $\zeta^{(1)}$ needs the second (multiplied by $k^{2}$ ) and the third. We have used the expressions (21) and (22) for $\delta p^{(0)}$ and $\xi^{(0)}$. By the symbols $F_{n}, G_{n}, J_{n}, K_{n}$ we generically indicate the results of all integrals involving the corresponding operators defined in Eqs. (21) and (22). Of course, these integrals, if present, are of order 1, according to the argument in Eq. (36). We will keep the symbols $F_{n}, G_{n}, J_{n}, K_{n}$ just to remember that these terms are present only if the corresponding operators are not zero. At the end it will be sufficient to put these symbols to 1 or 0 to consider models where these operators are present or absent, respectively.

In the second recursion we need the integrals

$$
\begin{aligned}
& \int_{-1}^{1} f_{\eta}(\eta) \zeta^{(1)} d \eta=\sum_{n=0}^{\infty}\left(F_{n} k^{-\nu+2 n}+G_{n} k^{\nu-2+2 n}+J_{n} k^{-\nu+2 n+2}+K_{n} k^{\nu+2 n}\right) \\
& \int_{-1}^{1} f_{\eta}(\eta) \xi^{(1)} d \eta=\sum_{n=0}^{\infty} K_{n} k^{-\nu+2 n}+\sum_{n, m=0}^{\infty}\left(J_{m} F_{n} k^{-\nu+2 m+2 n}+J_{m} G_{n} k^{\nu-2+2 m+2 n}\right. \\
& +J_{m} J_{n} k^{-\nu+2+2 m+2 n}+J_{m} K_{n} k^{\nu+2 m+2 n} \\
& \left.+K_{m} J_{n} k^{-\nu+2 m+2 n}+K_{m} K_{n} k^{\nu-2+2 m+2 n}\right) \\
& \int_{-1}^{1} f_{\eta}(\eta) \delta p^{(1)} d \eta=\sum_{n=0}^{\infty} G_{n} j^{-\nu+2 n}+\sum_{n, m=0}^{\infty}\left(F_{m} F_{n} k^{-\nu+2 m+2 n}+F_{m} G_{n} k^{\nu-2+2 m+2 n}\right.
\end{aligned}
$$




$$
\begin{aligned}
& +F_{m} J_{n} k^{-\nu+2+2 m+2 n}+F_{m} K_{n} k^{\nu+2 m+2 n} \\
& \left.+G_{m} J_{n} k^{-\nu+2 m+2 n}+G_{m} K_{n} k^{\nu-2+2 m+2 n}\right) .
\end{aligned}
$$

These integrals are those used in Sect. 6.3 to derive the post-bounce solution for scalar perturbations.

\section{References}

[1] Veneziano G, 1991 Phys. Lett. B 265, 287

Gasperini M and Veneziano G, 1993 Astropart. Phys. 1, 317

Gasperini M and Veneziano G, 2003 Phys. Rep. 373, 1

[2] Constantinidis C P, Fabris J C, Furtado R G and Picco M, 2000 Phys. Rev. D 61, 043503

Kogan I I, Mouslopoulos S, Papazoglou A and Ross G G, 2001 Phys. Rev. D 64, 124014

Mukherji S and Peloso M, 2002 Phys. Lett. B bf 547, 297

Kachru S and McAllister L, 2003 JHEP 0303, 018

Shtanov Y and Sahni V, 2003 Phys. Lett. B 557, 1

Foffa S, 2003 Phys. Rev. D 68, 043511

Veneziano G, 2004 JCAP 0403, 004

Setare M R, 2004 Phys. Lett. B bf 602, 1

Date G and Mortuza Hossain G, 2005 Phys. Rev. Lett. 94, 011302

Rinaldi M and Watts P, 2005 JCAP 0503, 006; Herdeiro C A R and Sampaio M, 2005 Preprint hep-th/0510052

Carloni S, Dunsby P K S and Solomons D, 2005 Preprint gr-qc/0510130

[3] Khouri J, Ovrut B A, Steinhardt P J and Turok N, 2002 Phys. Rev. D 66, 046005

Gratton S, Khoury J, Steinhardt P J and Turok N, 2004 Phys. Rev. D 69, 103505

Tolley A J, Turok N and Steinhardt P J, 2004 Phys. Rev. D 69, 106005

[4] Finelli F and Brandenberger R, 2002 Phys. Rev. D 65, 103522

[5] Biswas T, Mazumdar A and Siegel W, 2005 Preprint hep-th/0508194

[6] Brustein R, Gasperini M, Giovannini M, Mukhanov V and Veneziano G, 1995 Phys. Rev. D 51, 6744

[7] Enqvist K and Sloth M S, 2002 Nucl. Phys. B 626, 395

Lyth D H and Wands D, 2002 Phys. Lett. B 524, 5

Bozza V, Gasperini M, Giovannini M and Veneziano G, 2002 Phys. Lett. B 543, 14

Bozza V, Gasperini M, Giovannini M and Veneziano G, 2003 Phys. Rev. D 67, 063514

[8] Lyth D, 2002 Phys. Lett. B 524, 1

Brandenberger R and Finelli F, 2001 JHEP 0111, 056

Lyth D, 2002 Phys. Lett. B 526, 173

Hwang J, 2002 Phys. Rev. D 65, 063514; Tsujikawa S, 2002 Phys. Lett. B 526, 179

Martin J, Peter P, Pinto-Neto N and Schwarz D J, 2002 Phys. Rev. D 65, 123513

Hwang J and Noh H, 2002 Phys. Lett. B 545, 207

Martin J, Peter P, Pinto-Neto N and Schwarz D J, 2003 Phys. Rev. D 67, 028301

Tsujikawa S, Brandenberger R and Finelli F, 2002 Phys. Rev. D 66, 083513

Cartier C, Durrer R and Copeland E, 2003 Phys. Rev. D 67, 103517

Peter P, Pinto-Neto N and Gonzalez D A, 2003 JCAP 0312, 003

Creminelli P, Nicolis A and Zaldarriaga M, 2005 Phys. Rev. D 71, 063505 (2005)

[9] Durrer R and Vernizzi F, 2002 Phys. Rev. D 66, 083503

[10] C. Cartier, 2004 Preprint hep-th/0401036

[11] Gasperini M, Giovannini M and Veneziano G, 2003 Phys. Lett. B 569, 113

Gasperini M, Giovannini M and Veneziano G, 2004 Nucl. Phys. B 694, 206

[12] Allen L and Wands D, 2004 Phys. Rev. D 70, 063515 
[13] Bozza V and Veneziano G, 2005 Phys. Lett. B 625, 177

Bozza V and Veneziano G, 2005 JCAP 0509, 007

[14] Peter P and Pinto-Neto N, 2002 Phys. Rev. D 66, 063509

[15] Finelli F, 2003 JCAP 0310, 011

[16] Battefeld T J and Geshnizjani G, 2005 Preprint hep-th/0503160

[17] Hwang J and Noh H, 2002 Phys. Rev. D 65, 124010

Gordon C and Turok N, 2003 Phys. Rev. D 67, 123508

Martin J and Peter P, 2003 Phys. Rev. D 68, 103517

Martin J and Peter P, 2004 Phys. Rev. Lett. 92, 061301

Martin J and Peter P, 2004 Preprint gr-qc/0406062

[18] Deruelle N, 2004 Preprint gr-qc/0404126

Deruelle N and Streich A, 2004 Phys. Rev. D 70, 103504

[19] Tsujikawa S, private communication, reporting A. Starobinsky's opinion

[20] Battefeld T J, Patil S P and Brandenberger R H, 2005 Preprint hep-th/0509043

[21] Giovannini M, 2005, Class. Quant. Grav. 22, 2201

[22] Giovannini M, 2004, Phys. Rev. D 70, 103509 